\title{
Percepción de los estudiantes sobre el quehacer docente
}

\section{Perception of the students about the teaching task}

\author{
MARTÍNEZ-CÁRDENAS, Juana María†*, GARCÍA-GARCÍA, Jesús Alberto, LÓPEZ-NIEBLA, \\ Rosa María y TERRAZAS-MEDINA, Tamara Isabel
}

Universidad Autónoma de Coahuila, Instituto de Enseñanza Abierta, Unidad Saltillo. Campos Redondos SN, Sin Nombre de Col 9, 25000 Saltillo, Coah.

ID $1^{\text {er }}$ Autor: Juana María, Martínez-Cárdenas / ORC ID: 0000-0003-1004-9652, Researcher ID Thomson: X-23702018, CVU CONACYT ID: 949979

ID $1^{\text {er }}$ Coautor: Jesús Alberto, García-García / ORC ID: 0000-0003-1369-311X, Researcher ID Thomson: D-8211-2019, CVU CONACYT ID: 568438

ID $2^{\text {do }}$ Coautor: Rosa María, López-Niebla / ORC ID: 0000-0001-7260-3300, arXiv Author: 1908.09936, CVU CONACYT ID: 53192

ID $3^{\text {er }}$ Coautor: Tamara Isabel, Terrazas-Medina / ORC ID: 0000-0002-6581-190X, arXiv Author: 1908.09839, CVU CONCAYT ID: 929839

\begin{abstract}
Resumen
Los cambios actuales dan lugar a una nueva realidad social, familiar, económica, política y escolar, está ultima es el resultado de elementos multifactoriales que han cambiado la dinámica de trabajo en las aulas, la disiciplina, el ambiente escolar y las formas de aprendizaje. Por lo que el docente debe ser capaz de utilizar estrategias didácticas capaces de desarrollar habilidades en los estudiantes para que respondan a la diversidad y heterogeniedad de la sociedad de hoy. El objetivo de este estudio fue identificar las estrategias didácticas utilizadas por los docentes de bachillerato que propician aprendizajes significativos, acordes al saber aprender, saber hacer, saber ser y saber convivir, y describir las características de un buen docente. Se realizó una investigación cuantitativa de corte transversal que se aplicó a 126 estudiante de bachillerato, el $41.7 \%$ fueron hombres y el 57.5 fueron mujeres. La selección de la muestra fue por oportunidad. Los resultados muestran que las estrategias que más utililizan son la actitud positiva, la exposición, el compromiso, el dinamismo y el trabajo colaborativo como estrategias en su didáctica. Las características que más destacan en un buen maestro son: capacidad para enseñar, inteligente, trabajador, estricto honesto y responsable.
\end{abstract}

Estrategias didácticas, aprendizaje significativo y Educación Media Superior

Citación: MARTÍNEZ-CÁRDENAS, Juana María, GARCÍA-GARCÍA, Jesús Alberto, LÓPEZ-NIEBLA, Rosa María y TERRAZAS-MEDINA, Tamara Isabel. Percepción de los estudiantes sobre el quehacer docente. Revista de Educación Básica. 2019. 3-7: 27-34.

\begin{abstract}
Current changes lead to a new social, family, economic, political and educational reality, the educational one is the result of multifactorial elements that have changed the dynamics of classroom work, the discipline, the school environment and ways of learning. So the teacher should be able to use teaching strategies able to develop skills in students to respond to the diversity and heterogeneity of today's society. The goal of this study was to identify the teaching strategies used by high school teachers that promote significant learning chords to learn to learn, knowhow, know how to be and how to live, and describe the characteristics of a good teacher. A quantitative crosssectional research was applied to 126 high school students; $41.7 \%$ were male and $57.5 \%$ were women. The sample selection was by chance. The results show that the strategies that are the most used by the teachers are: positive attitudes, persuasion, commitment, exposition and collaborative work. The features that stand out in a good teacher are: ability to teach, intelligent, hardworking, strict, honest and responsible
\end{abstract}

Strategies, Meaningful Learning, School Education

*Correspondencia al Autor (Correo Electrónico: juanita_martinez17@ @otmail.com)
$\dagger$ Investigador contribuyendo como primer autor. 


\section{Introducción}

La educación es un sistema complejo de elementos que interactúan entre sí para formar al hombre y desarrollar sus habilidades, sin embargo, esto no es un proceso simple, los problemas educativos de una sociedad son multifactoriales y deben ser resueltos desde raíz y para ello se debe analizar la estructura educativa y la participación de cada una de las personas involucradas en el acto de enseñanza. En este sentido el papel del docente es fundamental en el proceso de aprendizaje de los estudiantes y el éxito en los resultados dependerá de los métodos, estrategias, técnicas y herramientas que utiliza el maestro dentro y fuera del aula para incentivar a sus estudiantes y lograr sus aprendizajes.

Esta investigación surgió a partir de las siguientes preguntas ¿Qué estrategias didácticas utilizan los docentes para propiciar aprendizajes significativos en los estudiantes del Instituto de Ciencias y Humanidades Lic, González Lobo y el Instituto de Enseñanza Abierta Unidad Saltillo? ¿Qué estrategias de aprendizaje propuestas en el modelo educativo de la UAdeC utilizan los docentes del ICH y el IDEA? y ¿Cuáles son las características de un buen docente desde la percepción de los estudiantes del ICH y de IDEA de la Universidad Autónoma de Coahuila?

El objetivo de este estudio fue identificar las estrategias didácticas que utiliza el docente de bachillerato que propician aprendizajes significativos que contribuyen a la formación integral de los adolescentes y que son pertinentes a los pilares de la educación: saber aprender, saber hacer, saber ser y saber convivir. Además, se analizan las características idóneas de un buen maestro desde la perspectiva de los estudiantes. La educación es el motor de cambio de toda sociedad y por esto las metodologías de enseñanza deben ser otras. Lo único seguro en la sociedad del siglo XXI es la incertidumbre, es decir, el cambio permanente, un presente que no permanece y un estilo de vida líquido caracterizado por buscar lo inmediato, individualismo, placer por el placer y conectividad. Por lo anterior es importante comprender la importancia del docente en el proceso de aprendizaje-enseñanza y por ende descubrir cuales estrategias utiliza con eficiencia para lograr aprendizajes significativos en los estudiantes.
En este sentido Ballester (2002) menciona que el aprendizaje significativo es el acto en que los estudiantes se esfuerzan por conectar el nuevo conocimiento con el conocimiento que ya poseían y con lo que sucede en su actual entorno de aprendizaje. Además, la mayor parte del aprendizaje tiene lugar en un ambiente de colaboración. Esto permite que el estudiante se beneficie de las ayudar surgidas a raíz del diálogo con los compañeros y de las positivas consecuencias afectivas que se obtienen a través del éxito que supone dominar el nuevo conocimiento.

Cada conocimiento demanda estrategias de aprendizaje especializadas. Estas dos actividades analíticas necesarias para el aprendizaje significativo, la estructura cognoscitiva de los estudiantes y la naturaleza específica de los contenidos, se contemplan en el análisis cognitivo de la tarea, la teoría cognitiva, las neurociencias y el procesamiento de la información. Descomponer las habilidades y condiciones cognitivas de los aprendices durante la apropiación de los conocimientos y las habilidades puede constituir una cualidad de las acciones exitosas de los docentes. (Ponce, 2004). Es necesario unir, tejer, articular diversas perspectivas teóricas del aprendizaje para diferenciar los tipos de conocimientos y habilidades que deben obtener los aprendices, para conocer los estilos y las estructuras cognoscitivas de los estudiantes, indagar en sus conocimientos y habilidades previas, diseñar estrategias diferenciadas de acuerdo con sus estilos de aprendizaje y los diversos contenidos escolares, descomponer las tareas complejas, diagnosticar los niveles que alcanzan, ofrecer ayuda oportuna y cercana a sus necesidades, promover la reflexión y la colaboración entre iguales, diferenciar qué proceso de aprendizaje requieren la simple asociación de aquellos que demandan el desequilibrio y la reestructuración cognitiva; $y$, finalmente, para favorecer el clima cálido de colaboración y afecto con los alumnos. (Ponce, 2004)

Las teorías constructivistas del aprendizaje han señalado la necesidad de concebir al estudiante como un ser activo en la construcción del conocimiento, pero también replantea el papel del docente como agente activo en los procesos de construcción de los contenidos de enseñanza de sus estudiantes, así como en el diseño, operación y evaluación de los recursos, estrategias o actividades que contribuyan a su desarrollo.

MARTÍNEZ-CÁRDENAS, Juana María, GARCÍA-GARCÍA, Jesús Alberto, LÓPEZ-NIEBLA, Rosa María y TERRAZAS-MEDINA, Tamara Isabel. Percepción de los estudiantes sobre el quehacer docente. Revista de Educación Básica. 2019 
Por otro lado, Cobian, Solís y Nielsen, (1999) dan a conocer procesos de innovación de la práctica educativa a través de la incorporación de dispositivos para reconocer la zona de desarrollo próximo de los alumnos, noción de Vigotsky como se citó en Ponce, (2004) y el aprendizaje significativo en estudiantes de secundaria.

Concluyen que existen evidencias acerca de cómo ayudar a los docentes mediante las aportaciones de la teoría cognoscitiva y el enfoque sociocultural, que implica el despliegue de un nuevo papel y una reconceptualización de la propia práctica educativa.

Asimismo, la actividad académica no se realiza de forma impersonal, sino en un contexto social en que las relaciones entre profesores y alumnos pueden afectar al grado de aceptación personal y afecto que éstos experimentan de parte de aquellos.

Todo alumno busca sentirse aceptado como es. Por ello, si por la razón que sea un alumno experimenta rechazo por parte del profesor o, simplemente, que éste prefiere y trata de favorecer a los demás más que a él mismo, si puede procurará evitar la situación y, si no puede, se sentirá obligado, lo que no favorece en modo alguno su motivación por aprender (Alonso Tapia, 1992).

El conjunto de consecuencias que se derivan del distinto significado que la actividad académica puede tener para los alumnos ha llevado a analizar las consecuencias potenciales que se derivan de sus modos de actuación del profesor en relación con las distintas metas que persiguen los alumnos para crear entornos de aprendizaje que estimulen el interés y el esfuerzo de éstos por aprender (Alonso Tapia, 1991, 1997; Ames, 1992).

No obstante, como se señaló al comienzo, la motivación depende no sólo del significado de la actividad, sino también de saber cómo afrontar las tareas de aprendizaje y, en particular, las dificultades con que se encuentran.
Realmente es difícil conceptualizar el término de pensamiento crítico, a pesar de que se encuentra presente en las metas o intenciones educativas de muchos proyectos curriculares. Sobre todo, en relación con los aprendizajes vinculados con la historia, el civismo o en los recientes programas de educación en moral o valores, suele encontrarse la afirmación que lo que se busca con su estudio es la formación de alumnos críticos, lo cual suele equipararse a que tomen conciencia o cuestionen su realidad social e histórica y participen activamente en su papel de actores sociales. (Díaz, Barriga, 2001) El aprendizaje significativo comporta también estrategias por descubrimiento, sobre todo para la adquisición de conceptos previos o relevantes. Luego la diferenciación e incorporación de otros conceptos a las estructuras cognitivas podrá efectuarse sin ningún problema por asimilación transmisión recepción o mediante la combinación de descubrimiento y asimilación.

El aprendizaje por descubrimiento es aquel que se preocupa más por la organización de la información que por su almacenamiento y acumulación. Considera que la mejor forma de asimilar de modo significativo una información es descubriéndola a través de la experiencia personal. Al alumno, no se le deben proporcionar contenidos ya elaborados, tal como se hace por el método de transmisiónrecepción, sino que se le deben dar las claves e instrumentos necesarios para que los descubra. Es el propio alumno quien debe comprobar personalmente la lógica de la respuesta antes de construirla y quien tiene que situarla correctamente en su propia estructura de conocimiento a través de los diversos niveles de representación. (Zárate, 1995)

\section{La aproximación constructivista, del aprendizaje y la enseñanza}

Hoy en día no basta con hablar del reconstructivismo en singular, es necesario decir a qué constructivismo nos estamos refiriendo. Es decir, hace falta el contexto de origen, teorización y aplicación de los mismos. En realidad, nos enfrentamos a una diversidad de posturas que pueden caracterizarse genéricamente como constructivistas. 
El constructivismo es una corriente pedagógica basada en la teoría del conocimiento constructivista, que postula la necesidad de entregar al alumno herramientas (generar andamiajes) que le permitan crear sus propios procedimientos para resolver una situación problemática, lo cual implica que sus ideas se modifiquen y siga aprendiendo.

El constructivismo educativo propone un paradigma en donde el proceso de enseñanza se percibe y se lleva a cabo como un proceso dinámico, participativo e interactivo del sujeto, de modo que el conocimiento sea una auténtica construcción operada por la persona que aprende (por el "sujeto cognoscente"). El constructivismo en pedagogía se aplica como concepto didáctico en la enseñanza orientada a la acción.

El constructivismo considera al alumno poseedor de conocimientos sobre los cuales habrá de construir nuevos saberes. Según Ausubel "Sólo habrá aprendizaje significativo cuando lo que se trata de aprender se logra relacionar de forma sustantiva y no arbitraria con lo que ya conoce quien aprende, es decir, con aspectos relevantes y preexistentes de su estructura cognitiva". A partir de los conocimientos previos de los educandos, el docente guía para que los estudiantes logren construir conocimientos nuevos y significativos, siendo ellos los actores principales de su propio aprendizaje. Por otra parte, el humanismo se refiere al estudio y promoción de los procesos integrales de la persona. La personalidad humana es una organización o totalidad que está en continuo proceso de desarrollo y la persona debe ser estudiada en su contexto interpersonal y social.

Desde el punto de vista humanista, la educación se debe centrar en ayudar a los alumnos para que decidan lo que son y lo que quieren llegar a ser. La educación humanista tiene la idea de que los alumnos son diferentes y los ayuda a ser más como ellos mismos y menos como los demás. La educación humanista es de tipo indirecto, ya que el docente permite que los alumnos aprendan impulsando y promoviendo todas las exploraciones, experiencias y proyectos, que estos preferentemente inicien o decidan emprender y logren aprendizajes vivenciales con sentido.
El paradigma humanista considera a los alumnos como entes individuales, únicos y diferentes de los demás. Son seres con iniciativa, con necesidades personales de crecer, con potencialidad para desarrollar actividades y solucionar problemas creativamente. Los alumnos no son seres que únicamente participen cognitivamente sino personas que poseen afectos, intereses y valores particulares y se les debe considerar como personas totales.

Los rasgos que debe tomar el humanista Son:

a. Ser un maestro interesado en el alumno como persona total.

b. Procurar estar abierto a nuevas formas de enseñanza.

c. Fomentar el espíritu cooperativo.

d. Ser auténtico y genuino ante los alumnos.

e. Intentar comprender a sus alumnos poniéndose en su lugar (empatía) y ser sensible a sus percepciones y sentimientos.

f. Rechazar las posturas autoritarias y egocéntricas.

g. Poner a disposición de los alumnos sus conocimientos y experiencias y que cuando lo requieran puedan contar con ellos.

\section{Metodología}

Participantes.

El tipo de muestreo que se utilizó en esta investigación fue por oportunidad, estuvo compuesto por 127 adolescentes de educación media superior, de dos preparatorias que pertenecen a la Universidad Autónoma de Coahuila. Los participantes tienen edades que oscilan entre los 15 y 24 años que viven en las ciudades de Saltillo y Acuña, Coahuila, México. Respecto a las edades el $22.83 \%$ reportó tener 15 años, el $44.09 \%$ especificó tener 16 años, el $21.25 \%$ reportó tener 17 años, el $6 \%$ dijo tener 18 años, el $2.03 \%$ dijo tener 19 años y el menos del $1 \%$ reportó tener 20 años y el $1.5 \%$ respondió tener 24 años. 
La aplicación de las encuestas se realizó a partir del consentimiento informado que fue otorgado por los docentes responsables de los grupos de cada Institución. Las escuelas que participaron son: Instituto de Ciencias y Humanidades Lic, González Lobo y el Instituto de Enseñanza Abierta Unidad Saltillo.

\section{Instrumento.}

Para la recolección de datos en los estudiantes se utilizó una encuesta de elaboración propia, el cuestionario consta de 33 ítems los cuales reportaron fiabilidad, esto se calculó a través del método alfa de Cronbach y se obtuvo un valor de .90 , esto indica un alto nivel de consistencia interna del instrumento (Hernández, Fernández y Collado, 2014). En 27 de los 33 reactivos se utilizó escala ordinal tipo Likert. La forma de respuesta fue de 5 opciones adolescente respecto a las situaciones que le son presentadas. El valor asignad para cada pregunta va desde 0 para cuando se está totalmente en desacuerdo de izquierda a derecha, 1 cuando se está en desacuerdo, 2 ni de acuerdo ni en desacuerdo, 3 de acuerdo y hasta 4 para totalmente de acuerdo. Los primeros Cinco datos fueron indicadores sociodemográficos (edad, sexo, promedio).

\section{Procedimiento.}

Con la información obtenida en la administración del instrumento, se construyó una matriz de datos en el programa estadístico SPSS versión 19. El análisis de las estrategias didácticas y el aprendizaje significativo se llevó a cabo a partir de la estadística descriptiva y prueba $t$ para muestras independientes, para identificar las características de un buen docente, se realizó a partir de la estrategia de análisis multivariado análisis de cluster.

\section{Resultados}

Con el propósito de identificar las estrategias de aprendizaje más utilizadas por los docentes de bachillerato, se realizó el análisis estadístico de los datos a través de la estadística descriptiva, los índices que se usaron son: Media, puntaje mínimo y máximo, desviación típica, sesgo y curtosis. que corresponden a la forma de sentir del

En promedio los docentes utilizan como estrategias de aprendizaje la actitud positiva, la exposición, el compromiso, el dinamismo y el trabajo colaborativo, estas variables obtuvieron valores en la media mayores a 3 . El puntaje mínimo fue de 0 y el puntaje máximo fue de 4 , lo que indica que en estar variables los participantes utilizaron toda la escala. De acuerdo al sesgo se observó que la mayoría de las variables tienen valores negativos lo que indica que hay una concentración de los datos en los valores altos de la escala.

\begin{tabular}{|l|r|r|r|r|r|r|r|}
\hline Variables & \multicolumn{1}{l}{$\mathbf{n}$} & \multicolumn{1}{l}{ Mín } & \multicolumn{1}{l}{ Máx } & \multicolumn{1}{l}{ DE } & \multicolumn{1}{l}{ AS } & \multicolumn{1}{l|}{ K } \\
\hline $\begin{array}{l}\text { Actitud } \\
\text { positiva }\end{array}$ & 126 & 3.46 & 0 & 4 & .786 & -1.722 & 3.462 \\
\hline Exposición & 125 & 3.22 & 0 & 4 & .857 & -1.213 & 1.934 \\
\hline Compromiso & 125 & 3.21 & 0 & 4 & .806 & -1.242 & 2.675 \\
\hline $\begin{array}{l}\text { Trabajo } \\
\text { colaboración }\end{array}$ & 125 & 3.21 & 0 & 4 & .796 & -.880 & .981 \\
\hline $\begin{array}{l}\text { Dinámicas de } \\
\text { trabajo }\end{array}$ & 126 & 3.20 & 0 & 4 & .820 & -.914 & .903 \\
\hline Innovación & 126 & 3.15 & 1 & 4 & .749 & -.603 & .077 \\
\hline $\begin{array}{l}\text { Ambientes de } \\
\text { aprendizaje }\end{array}$ & 126 & 3.14 & 1 & 4 & .734 & -.354 & -.663 \\
\hline Creatividad & 123 & 3.06 & 0 & 4 & .813 & -.850 & 1.105 \\
\hline Saber convivir & 124 & 3.06 & 1 & 4 & .789 & -.605 & .087 \\
\hline Saber ser & 126 & 3.01 & 0 & 4 & .805 & -.950 & 1.943 \\
\hline Lúdica & 126 & 2.99 & 0 & 4 & 1.054 & -.982 & .446 \\
\hline Investigación & 126 & 2.93 & 0 & 4 & .878 & -1.157 & 1.934 \\
\hline Saber hacer & 124 & 2.92 & 0 & 4 & .852 & -.487 & .088 \\
\hline $\begin{array}{l}\text { Aprendizaje } \\
\text { basado en } \\
\text { proyectos }\end{array}$ & 125 & 2.90 & 0 & 4 & .928 & -.790 & .762 \\
\hline Saber aprender & 125 & 2.90 & 0 & 4 & .777 & -.355 & .365 \\
\hline Saber convivir & 123 & 2.89 & 1 & 4 & .802 & -.276 & -.446 \\
\hline
\end{tabular}

Tabla 1 Estadística descriptiva de la variable estrategias de aprendizaje

Asimismo, se realizó la estadística descriptiva de la variable compleja aprendizaje significativo, se observó que en promedio los estudiantes construyen su aprendizaje a partir de la comprensión y descubrimiento de los temas, asumen la responsabilidad de su aprendizaje y realizan trabajos integrando información de diversos temas.

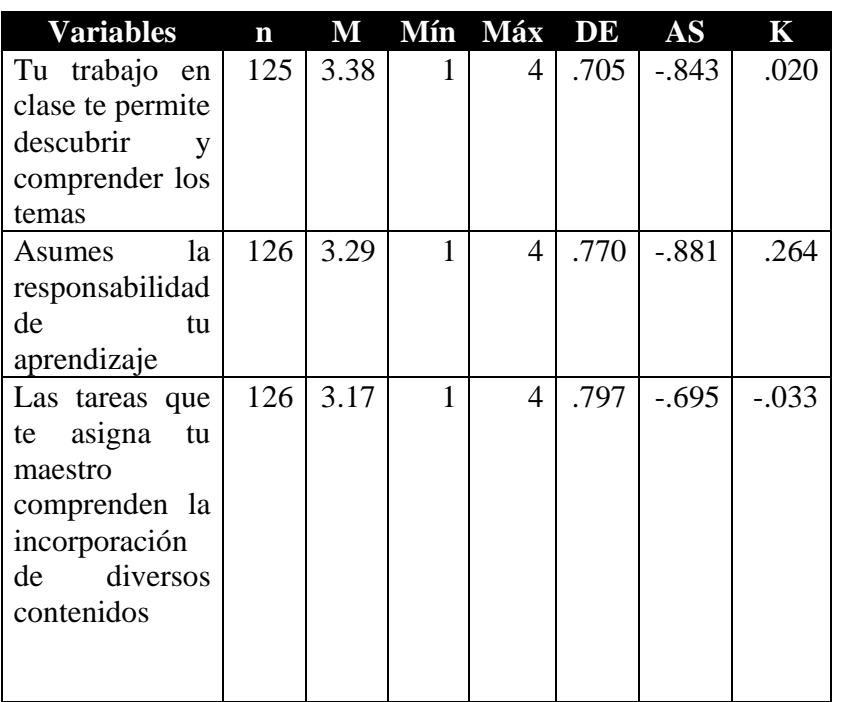

MARTÍNEZ-CÁRDENAS, Juana María，GARCÍA-GARCÍA，Jesús Alberto, LÓPEZ-NIEBLA, Rosa María y TERRAZAS-MEDINA, Tamara Isabel. Percepción de los estudiantes sobre el quehacer docente. Revista de Educación Básica. 2019 


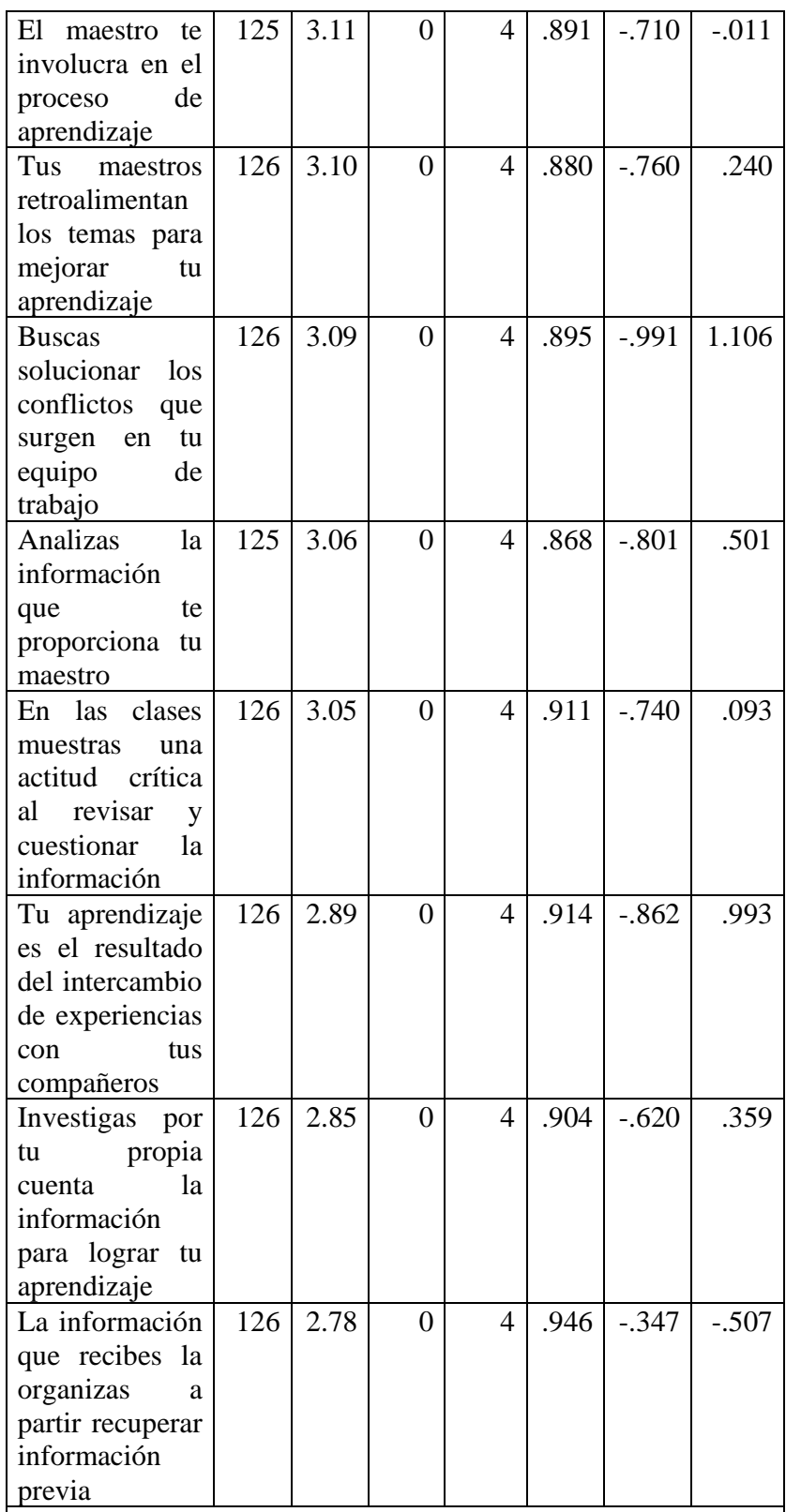

Nota: $\mathrm{n}=$ muestra, $\mathrm{M}=$ media, Mín= puntaje mínimo, Máx= puntaje máximo, $\mathrm{DE}=$ desviación típica, $\mathrm{AS}=$ sesgo, $\mathrm{K}=$ curtosis.

Tabla 2 Estadística descriptiva de la variable aprendizaje significativo

Con el propósito de determinar las diferencias por sexo en la percepción de las estrategias de aprendizaje que utilizan los docentes, se procesó una prueba t para muestras independientes. Para asegurarse de que no exista un incumplimiento extremo de los supuestos de la prueba, se obtuvo la prueba de Homogeneidad de las varianzas, la que aceptó la hipótesis nula de homocedasticidad. El nivel de significancia utilizado para la prueba de hipótesis fue de $\mathrm{p} \leq .050$. Hay diferencias significativas por sexo en la variable saber aprender y saber convivir, la media fue mayor en el grupo de mujeres, el valor de esta variable se encuentra a un punto del valor máximo posible para esta escala.
Esto demuestra que las mujeres, aplican lo aprendido en clase en función de su contexto social más que los hombres y tienen mayor participación en la comunidad con respecto a los hombres.



Tabla 3 Análisis comparativo por sexo sobre estrategias de aprendizaje por la prueba $t$ para muestras independientes

Por otra parte, se realizó un análisis comparativo por escuela para encontrar las diferencias significativas entre el Bachillerato escolarizado y el bachillerato escolarizado a distancia respecto al aprendizaje significativo, se encontró que existen diferencias significativas en cuanto al involucramiento del estudiante en el proceso de aprendizaje, con una media mayor en el Bachillerato escolarizado que el bachillerato a distancia. Se observó una actitud más crítica y participativa en los estudiantes del bachillerato escolarizado que el bachillerato a distancia.

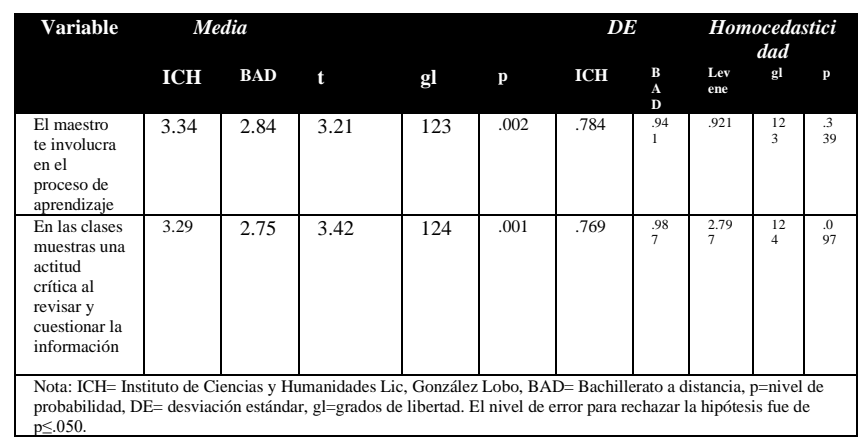

Tabla 4 Análisis comparativo por Escuela del factor aprendizaje significativo por la prueba $t$ para muestras independientes 
El siguiente gráfico muestra el análisis de cluster del ítem, características de un buen docente, en este sentido se pudo observar tres grandes agrupaciones respecto a las cualidades de un buen docente; el bloque uno contiene características asociadas a la parte actitudinal del docente, es decir, amable, atento, comprensivo y paciente, el bloque dos refiere a cualidades axiológicas, es decir, honestidad y responsabilidad, y el bloque tres refiere la parte profesional, es decir, capacidad de enseñar, trabajador, disciplina e intelectualidad.

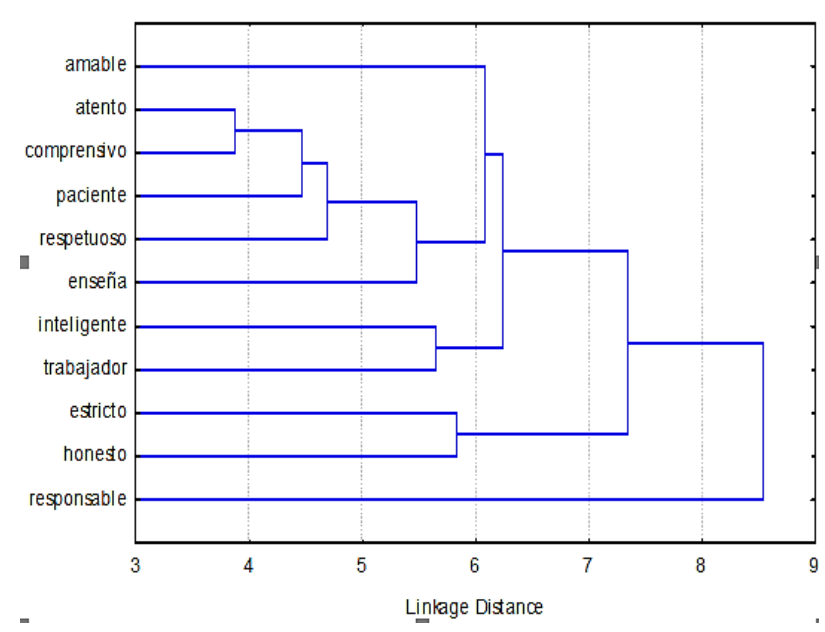

Gráfico 1 Cluster análisis. Características del docente. Euclidean distances

\section{Conclusiones}

El trabajo docente es una actividad compleja y polifacética, es decir, educar a una persona es todo un proceso que implica la articulación de un conjunto de factores relacionados con el acto educativo. Por lo anterior es importante identificar las estrategias, métodos, técnicas, recursos que utilizan los docentes del bachillerato que propician aprendizajes significativos en los estudiantes. Se concluye que las principales estrategias de aprendizaje que utilizan los docentes son: trabajo colaborativo, trabajo en equipo y ambientes de aprendizaje que involucran la creatividad, innovación, dinamismo y actitud positiva.

Lo anterior permite decir que las estrategias didácticas que utiliza el docente son un medio fundamental para formar integralmente a los adolescentes, el aprendizaje es el resultado de la realización del saber aprender, hacer, ser y convivir para lograr que los estudiantes tengan la posibilidad de tener éxito en la vida.
Respecto a lo que los estudiantes refieren del significado de ser un buen maestro se pudo concluir que son 11 atributos que describen esta categoría. En este sentido los estudiantes piensan que un buen docente es aquel que: es amable, atento, comprensivo, paciente, respetuoso, tiene capacidad para enseñar, inteligente, trabajador, estricto que pone disciplina, honesto y responsable. Estas características permiten comparar la realidad del acto educativo con lo que el modelo educativo de la Universidad Autónoma de Coahuila (UAdeC) establece sobre los valores institucionales, tales como: honestidad, responsabilidad, libertad, justicia, respeto, solidaridad, tolerancia y compromiso. En este sentido se concluye que las características de un buen docente que son importantes para los estudiantes están acordes al Modelo educativo de la UAdeC (2014), ya que ésta basa su quehacer académico y su operación administrativa en el apego a los valores, que forman parte del código de Ética Institucional. $\mathrm{Se}$ concluye que existen diferencias significativas por sexo respecto a la manera en que se involucra el estudiante en su proceso de aprendizaje y en la actitud para revisar y cuestionar la información que presenta el docente, donde las mujeres tiene un nivel mayor que los hombres respecto a la participación y el pensamiento crítico. Las estrategias de aprendizaje que plantea el Modelo Educativo de la UAdeC, en su mayoría son utilizadas por los docentes de estos dos bachilleratos, estás son el aprendizaje basado en problemas, el trabajo colaborativo, el método de casos, aprendizaje basado en proyectos $\mathrm{y}$ desarrollo de habilidades del pensamiento.

\section{Referencias}

Angel D. López M., F. F. (2000). La Formación de Docentes en Física para el Bachillerato. Reporte y reflexión sobre un caso. Revista Mexicana de Investigación Educativa, 113-135.

Arceo, F. D. (1998). Una Aportación a la Didáctica de la Historia. La EnseñanzaAprendizaje de Habilidades Cognitivas en el Bachillerato. Perfiles Educativos, 82.

Barrig, F. D. (2001). Habilidades del pensamiento crítico sobre contenidos históricos en alumnos de bachillerato. Revist Mexicana de Investigación Educativa. 
Cervera, M. d. (2012). Estrategias de Aprendizaje Cooperativo para Alumnos de Bachillerato con Bajo Rendimiento Académico. Mérida.

Díaz Barriga, F. (2001). Habilidades de pensamiento crítico sobre contenidos históricos en alumnos de bachillerato. Revista Mexicana de Investigación Educativa, 6(13) Recuperado de

http://www.redalyc.org/articulo.oa?id=1400130 8

Emma Jiménez Gómez, M. d. (2001). La formación de formadores de bachillerato en sus propios centros docentes. Revista de Investigación y Experiencias Didácticas, 163170.

ENNA CARVAJAL CANTILLO, M. D. (2002). Concepciones y representaciones de los maestros de secundaria y bachillerato sobre la naturaleza, el aprendizaje y la enseñanza de las ciencias. Revista Mexicana de Investigación Educativa, 577-602.

Frida Díaz Barriga Arceo, G. H. (2002). Constructivismo y Aprendizaje Significativo, una interpretación constructivista. En G. H. Frida Díaz Barriga Arceo, Estrategias Docentes para un Aprendizaje Significativo (págs. 231249). México DF: Mc Graw Hill.

Guerrero, J. L. (1997). Estrategias para un Aprendizaje Significativo-Constructivista. Ediciones Universidad de Salamanca, 29-50.

Gutiérrez, O. Á. (2003). El Proceso Educativo desde los Enfoques Centrados en el Aprendizaje.

María Eugenia Martín Palacio, J. A. (2010). Evaluación del Aprendizaje Autoregulado en estudiantes de bachillerato mexicanos. Aula Abierta, 59-70.

Martín, A. Z. (1995). Aprendizaje significativo y geografía de las representaciones mentales. Revistas UCM, 831-840.

Moreira, M. (2010). ¿Por qué Conceptos? ¿Por qué Aprendizaje Significativo? ¿Por qué Actividades Colaborativas? ¿Por qué Mapas Conceptuales? Revista Qurriculum, 9-23.
Ponce, V. (2004). El aprendizaje significativo en la investigación educativa en Jalisco. Sinéctica24, 21-29.

Sahelices, M. C. (2009). ¿Qué aprendizaje promueve el desarrollo de competencias? una mirada desde el aprendizaje significativo. Revista de teoría, investigación y práctica educativa, 11-34.

Sergio Tobón Tobón, J. H. (2010). Secuencias Didácticas. Aprendizaje y Evvaluación de Competencias. México, D.F.: Pearson Education.

Tapia, J. A. (2005). Motivación para el aprendizaje: La perspectiva de los alumnos. Ministerio de Educación y Ciencia , 209-242.

Vallori, A. B. (2010). El Aprendizaje Significativo en la Práctica. España.

Vidales, S. (2009). El Fracaso Escolar en la Educación Media Superior. El caso de Bachillerato de un Universidad Mexicana. Revista Iberoamericana sobre Calidad, Eficacia y Cambio en Educación, 320-341 